\title{
Smart Security for an Organization based on IoT
}

\author{
Mohd. Saifuzzaman \\ Student \\ Department of Computer \\ Science \& Engineering \\ Daffodil International \\ University \\ Dhaka, Bangladesh
}

\author{
Ashraf Hossain Khan \\ Student \\ Department of Computer \\ Science \& Engineering \\ Daffodil International \\ University \\ Dhaka, Bangladesh
}

\author{
Nazmun Nessa \\ Moon \\ Assistant Professor \\ Department of Computer \\ Science \& Engineering \\ Daffodil International \\ University \\ Dhaka, Bangladesh
}

\author{
Fernaz Narin Nur \\ Assistant Professor \\ Department of Computer \\ Science \& Engineering \\ Daffodil International \\ University \\ Dhaka, Bangladesh
}

\begin{abstract}
This research describes a low cost and flexible security system which is basically based on Arduino with necessary interface to enable Internet and the control of power through Global System for Mobile Communication (GSM) \& Bluetooth module (HC-05). This paper consumed more real life interactions along with embedded software solutions. In this project a password is set for the access of all sensors, for this we use LCD Display and Keypad. Motion sensor, gas module, reed sensor, laser sensor, all the sensors are used to detect theft and unwanted occurrences. Control Panel Interface of Web Server and android voice control both are created to control of all lights of the organization and for the purpose of power savings. The proposed system requires minimum human intervention to control the system. This research ensures the safety of organization from unwanted occurrence and theft. The main contribution of this paper is that it not only helps to ensure the security of an organization but also energy efficient and time saving.
\end{abstract}

\section{Keywords}

IoT, Arduino Mega, GSM module, SMS, Android, Smartphone, PIR, Gas sensor.

\section{INTRODUCTION}

Now a days Internet of Things (IoT) is basically a fast growing technology. D. Giusto et.al [1] and Li Da Zuet.al [2] described that IoT is basically the combination of physical device, software, sensors, network, wired or unwired technology etc which is basically used to make an ecosystem of computing like collect and exchange data. In the Internet of Things applications, physical sensors collect different types of data from the field and transfer those data to the Internet [22], [23]. N. Sklavos et.al [3] explained the requirement for security makes many of us seek for alternative ways so as to safeguard their property. The aim of this paper is to develop IoT Based Security System of an Organization. In this paper, we are developing a system which is an integration of several mini system. This system generates Alerts/Alarms or take intelligent decisions using concept of IoT. In our project we have a tendency to use both Bluetooth and GSM to manage the system. Here Bluetooth is chosen with its appropriate ability to manage the organization from indoor and GSM for outside. Also, most of the present laptop computer or cell phones are come with inbuilt Bluetooth adapter. Through GSM, the user will effectively management and monitor the organization from far off by causation SMS. Here we also use different types of sensors (motion, gas, reed and laser) and methods so that the system can flexible but is not a common system. The main objective of this paper is to help people that will enable them to control their privacy and alert them in some immediate situations if any occurrences happen.

\section{PREVIOUS WORK}

There are several definitions of automation and security system available in the literature. G. Gu et.al [4] and $G$. Mingming et.al [5] mentioned that all GSM is one of the most widely used cellular technologies in the world. $C$. Felix et.al [6], C. Peijiang et.al [7] and B. S. Rao [8] explained that with the increase in the number of GSM subscribers, research and development is heavily supported in further investigating the GSM implementation. Then Rozita Teymourzadeh et.al [9] focused on their [4-8] thoughts and implemented the functionality of GSM protocol for saving the power such as control light, fan and other home appliance. R. Llamas et.al [10] discussed GSM based device control system with mobile application developed using the app inventor for android mobile phones targeting its large market which is able to be helpful for the masses. Then Mahesh N. Jivani et.al [11] used $R$. Llamas's idea and also has the same functionality as [9] but it includes app inventor for android mobile. But I think, here is no room for security preventing from theft or fire or unwanted situation which is basically the main fact in 21 st century. After that in our research, we are going to explore new ideas \& thoughts which is the combination of saving power and well security system of an organization. For this we are going to use not only GSM but also Bluetooth, web server and android apps to provide a secure organization. Ming Yan et.al [12] proposed and prototyped a new smart living system known as home lighting control system using Bluetooth based android smartphone. Then B. Murali Krishna et.al [13] followed Ming Yan's and represented Bluetooth based wireless home automation system using FPGA. Bluetooth technology, that aims to exchange information wirelessly in a range of short distance by providing a necessary platform to form convenience and controllability. But the fact is, it only works on a limited range but in security matter it also fails to form the long range platform. That's why in our research we use also use Bluetooth along with GSM for long range platform also. Nathan David et.al [14] explored same as the paper [13] but includes more sensors and functionality. It added email notification method but in my point of view we are not that much used to an email but we are pretty much used to in a SMS notification so in our project and paper we use this SMS notification system based on the necessity \& availability. Sirisilla Manohar et.al [15] proposed at planning a basic home automation application on internet through reading the topic of E-mail which is also focused on a single component and as we said before SMS interaction is more compatible than Email. Prof. R.S. Suryavanshi et.al [16] discussed an approach in which a model of Home Automation System using android and WLAN technology, which really 
offers simple and much awaited Automation Systems. After that Poonam B. Patil et.al [17] used Prof. R.S. Suryavanshi's ideas and proposed a system to control the home appliance using android apps and GSM. In our project we use nearly the same component but the differences is that we have used some additional sensors to protect our organization from unwanted occurrences, fire and theft. Moreover, S.Anusha et.al [18] described the main objective of home automation and security is to assist disabled and old aged those who will alter them to manage home appliances and alert them in critical situations supported android system methodology. V.Persis Priyanka et.al [19] described every person wants his house, industry etc. to be secured. This project describes a security system which will monitor an industry and residential. A PIR sensing element is interfaced to the controller to notice the presence of intruders and instantly captures the image using camera connected to controller and forwards through E-mail and conjointly a buzzer alert is given to intimate others. S. M. Anamul Haque et.al [20] proposed a system that controls the home appliances using the private computer. This system is developed by using the Visual Basic 6.0 as programming language and Microsoft voice engine components for speech recognition purpose. Appliances can be controlled by timer or by voice command. Following S. M. Anamul Haque's idea, Amrutha $S$ et.al [21] proposed a system that described the voice recognition method by which the home appliances can be controlled.

\section{SYSTEM DESCRIPTION}

To demonstrate the feasibility and effectiveness of this system, devices such as GSM, Bluetooth module, PIR motion reed sensor, gas sensor and laser sensors have been integrated with the proposed Security system for an Organization. This integration is basically done for the purpose of higher security to maintain an organization in a proper and secure way. This system has two main modules: the hardware interface module the software communication module (Web application \& Android application). The core component of the system is Arduino Mega 2560 microcontroller that is additionally capable of functioning as a micro web server and also the interface for all the hardware modules. All communication and controls during this system go through the microcontroller. As we have already said that our project is the integration of several mini projects to make more efficient, flexible, low cost and reliable security system. So the objectives of proposed system is divided into two parts

The first one is controlling the appliances of the organization and second is controlling the security of the organization.

\subsection{Control for appliances}

In this research, we also represent a home automation system (light, fan and other appliances) which is secured and flexible that is controlled using web server and an android app in a cell phone.

\subsection{Control for security}

In this research, the security system offer two effective features which are intrusion detection and fire detection. This system can be able to notify the authority as well as the security officers based on the occurrences.

\subsubsection{Intruder detection}

As we can see in figure 1 , if any intruder is detected by the motion sensor/ reed sensor/ laser sensor, the system will immediately notify the nearby police station as well as the authority.

\subsubsection{Fire detection}

As we can see in figure 1, if the MQ2 Gas module sense gas/smoke/fire, the system will be able to notify nearby fire service as well as the authority.

(Arduino, GSM, Bluetooth and Sensors) and also
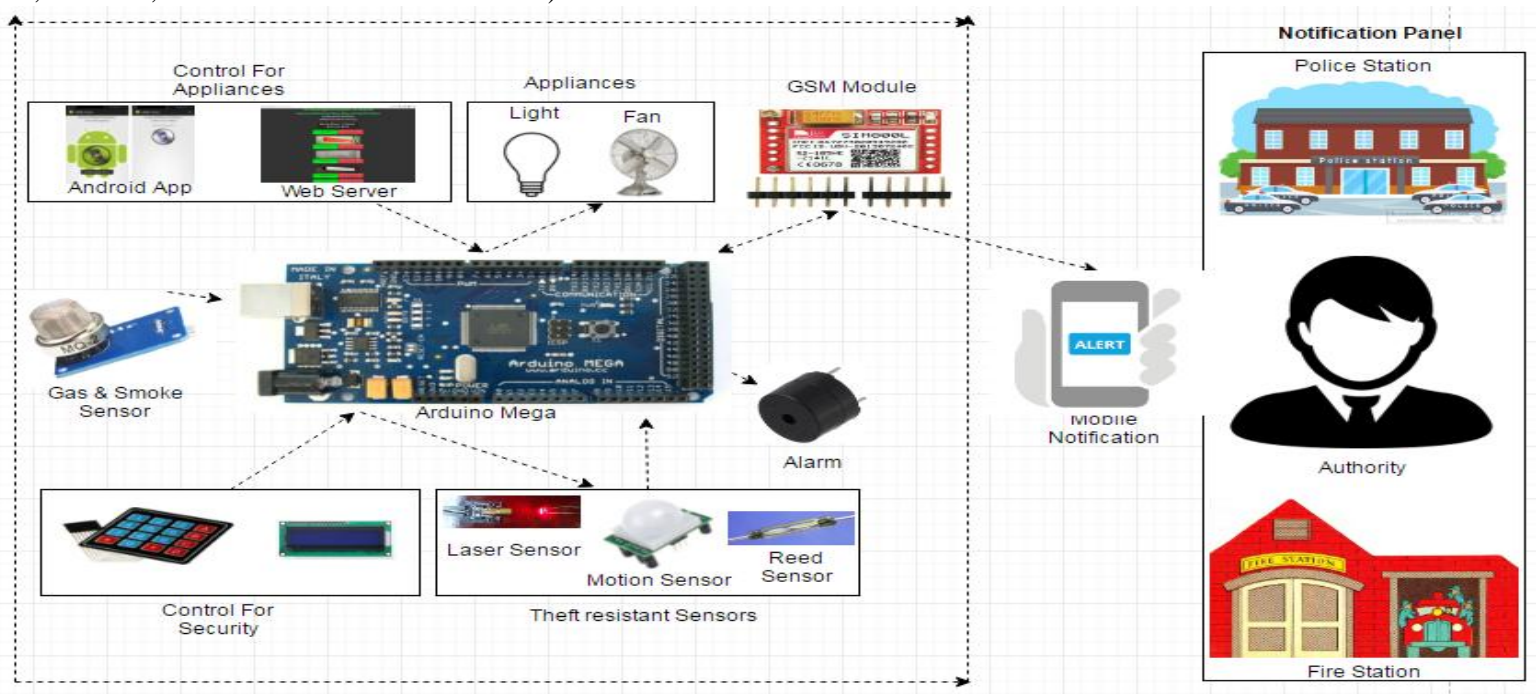

Figure 1: System Architecture of the Proposed System

\section{HARDWARE DESCRIPTION}

In this project, I have used various types of hardware which we are going to describe with details necessary features.

\subsection{Arduino (Mega2560)}

In Figure 2 the Arduino Mega is the driver behind our entire project, not only does it receive and interpret signals from our sensor devices, but it also allowed us to run the program entirely on it (lacking the benefits of the GUI and inexpensive cameras of course). Our reasoning for picking the Mega was that we knew for our prototype it would supply ample configurability and we would not have to be concerned with running out of connectors. This microcontroller uses $3.3 \mathrm{~V}$ and $5 \mathrm{~V}$ to power its related devices and is itself powered either by USB 500mA or can be powered via an external source up to $12 \mathrm{~V}$. We decided to power using USB partially due to convenience and also the added benefit of limiting harm done 
to the system by any mistakes we could potentially make in connections. The Mega is affordable at roughly sixty dollars.

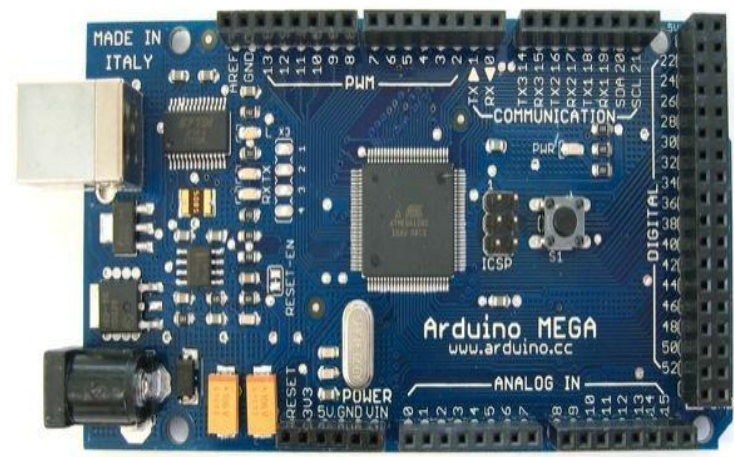

Figure 2: Arduino mega

\subsection{PIR Motion sensor}

This sensor is our typical passive infrared device. It detects motion at a distance of about 20 feet. This unit is relatively simple and has two output modes to choose from. We chose to use the high signal when motion was present. These have a retail cost of about ten dollars.
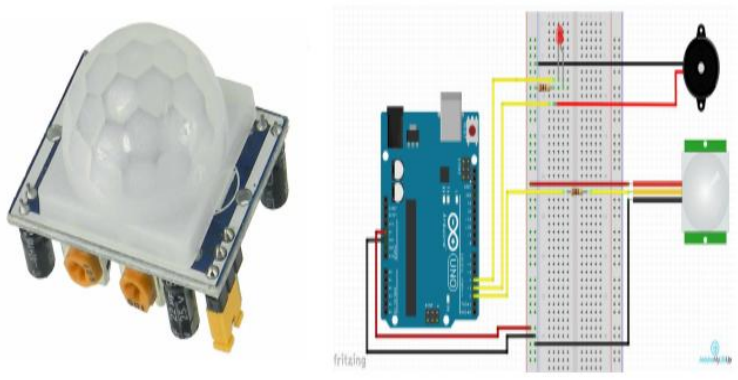

Figure 3: PIR (motion sensor)

\subsection{Smoke sensor (MQ2)}

In Figure 4 the MQ-2 Gas Sensor module is useful for gas leakage detecting in home and industry. It can detect LPG, ibutane, propane, methane, alcohol, hydrogen and smoke.
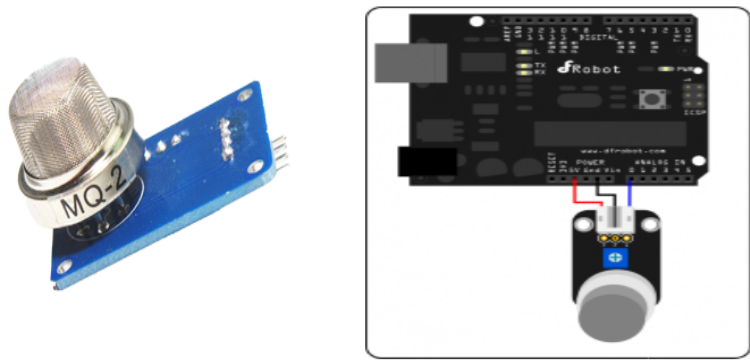

Figure 4: Smoke sensor

\subsection{Laser pointer \& photo Resister}

We set the laser pointer in such a way that the ray will be fall on to the photo resistor. If any intruder passes through the ray and when the ray doesn't fall on the photo resistor then the buzzer will active and make a sound.
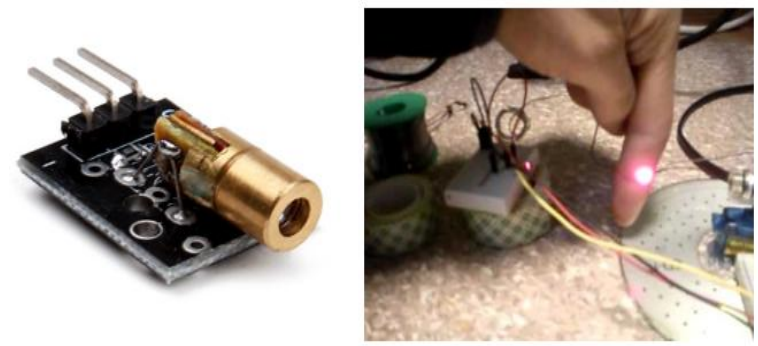

Figure 5: Laser pointer \& Photo Resister

\subsection{PIEZO buzzer}

In Figure 6 the buzzer or beeper is an audio signaling device which may be electromechanical, or piezoelectric. Typical uses of buzzers and beepers include alarm devices, timers, and confirmation of user input such as a mouse click or keystroke.
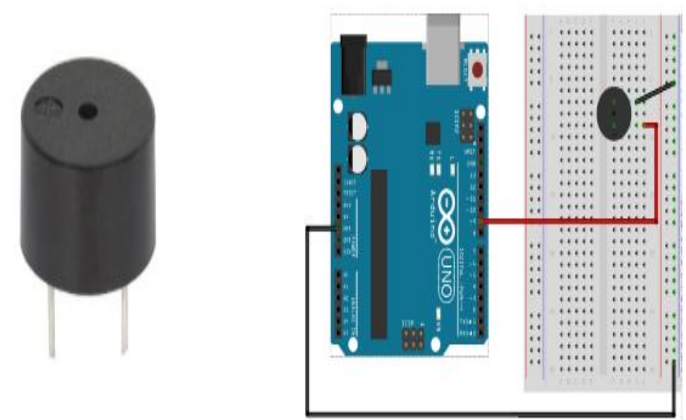

Figure 6: PIEZO buzzer

\subsection{SIM 800L core board}

In Figure 7 the SIM800 series modules support Hyper Text Transfer Protocol application. Which provides a mode to alternate of HTTP server. The basic application contains GET, POST, HEAD methods. SIM800 series modules support File Transfer Protocol application.

The Features are:

- Power supply: voltage range: $3.4 \mathrm{~V} 44.4 \mathrm{~V}$

- Power saving: SLEEP mode is $0.7 \mathrm{~mA}$ (BS-PAMFEMS=9).

- Band: GSM type: small mobile station

- Four frequency: EGSM900, DCS1800, GSM850, PCS1900, can automatically search the four frequency bands. You can also set the frequency band through the AT command.

- $\quad$ Accord GSM Phase $2 / 2+$

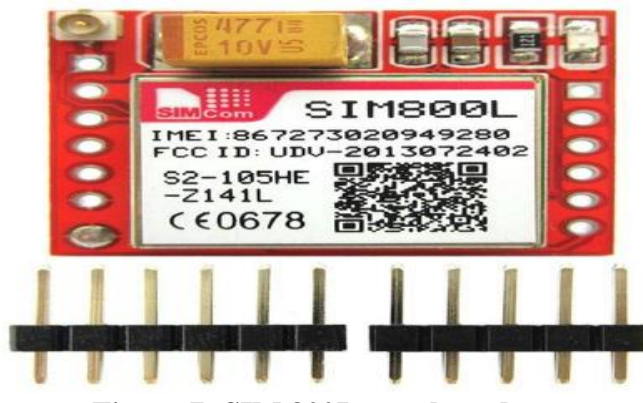

Figure 7: SIM 800L core board

\subsection{HC-05 serial port bluetooth}

In Figure 8 the following bluetooth is one of the popular wireless communication technologies because of its low power consumption, low cost and a light stack but 
compensates on range. The remote unit can be powered from $3.3 \mathrm{~V}$ up to $6 \mathrm{~V}$ for easy battery attachment. All signal pins on the remote unit are $3 \mathrm{~V}-6 \mathrm{~V}$ tolerant. No level shifting is required

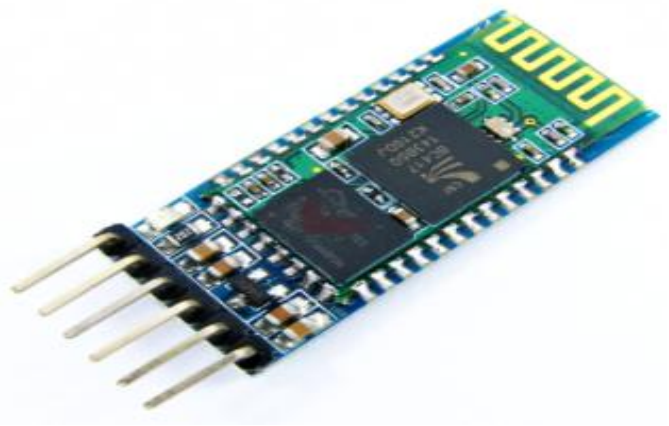

Figure 8: HC-05 Serial port Bluetooth

The specifications are:

- Bluetooth protocol: Bluetooth Specification v2.0+EDR

- Frequency: 2.4GHz ISM band

- Security: Authentication and encryption

- Profiles: Bluetooth serial port

- Power supply: +3.3VDC 50mA

- Power supply: +3.3VDC 50mA

- Working temperature: $-20 \sim+75$ Centigrade

- Dimensions: $15.2 \times 35.7 \times 5.6 \mathrm{~mm}$

\section{RESULT AND DISCUSSION}

At last project comes to a physical existence and looks as below. In Figure 9, here is the overall demonstration of our proposed system.

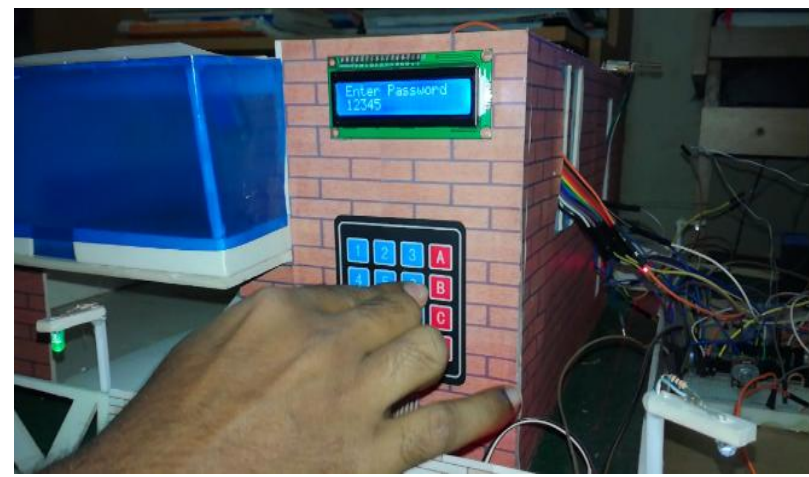

Figure 9: Overall demonstration of our proposed system.

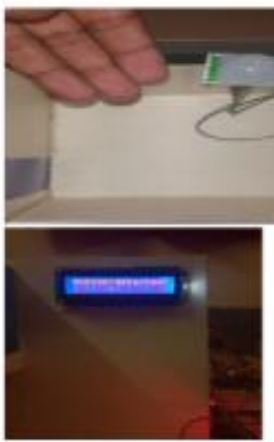

(a)
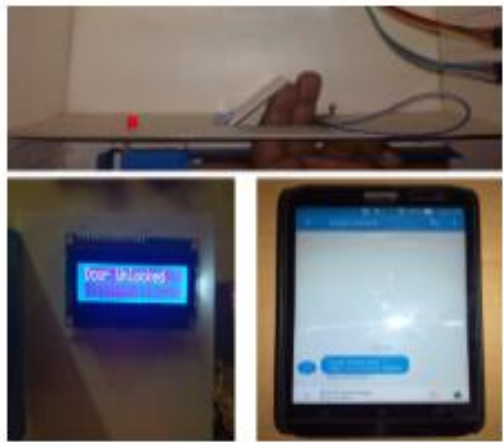

(b)

\subsection{Control for security}

This system has been accessed by secure password through keypad. Display will help to see the output. As following figures 10 shows how the system will work by password

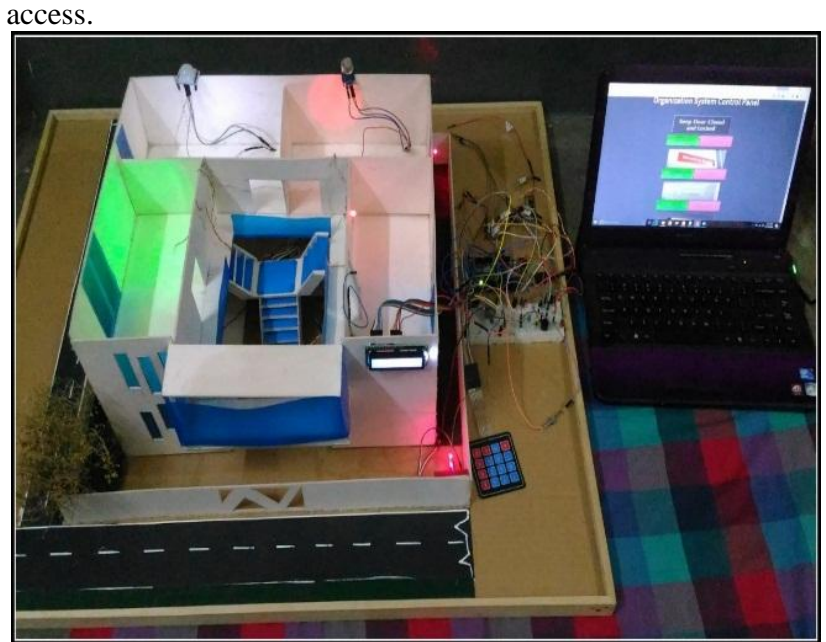

Figure 10: Access on the system by entering password through keypad

As the following figures 11(a) (b) (c), all show that live demo of proposed system. Here, (a) when movement detect around motion sensor (b) when door is unlocked by unauthorized people (c) someone passes through the laser, the system notifies through LED display and it makes a security alarm. After that, through GSM, it sends an SMS "Motion detected/Door Unlocked/Theft detected!!! Take Necessary Steps" to the authority as well as the police station.

After that, the following figure 12 shows when fire or gas detect around gas sensor, the system notifies through LED display and make a security alarm after that through GSM, it sends a SMS "Fire Fire!!! Take Necessary Steps" to the authority as well as the fire station.

Figure 11: (a) Live demo-motion detection (b) Live demo-Reed Sensor (c) Live demo-Laser Sensor 

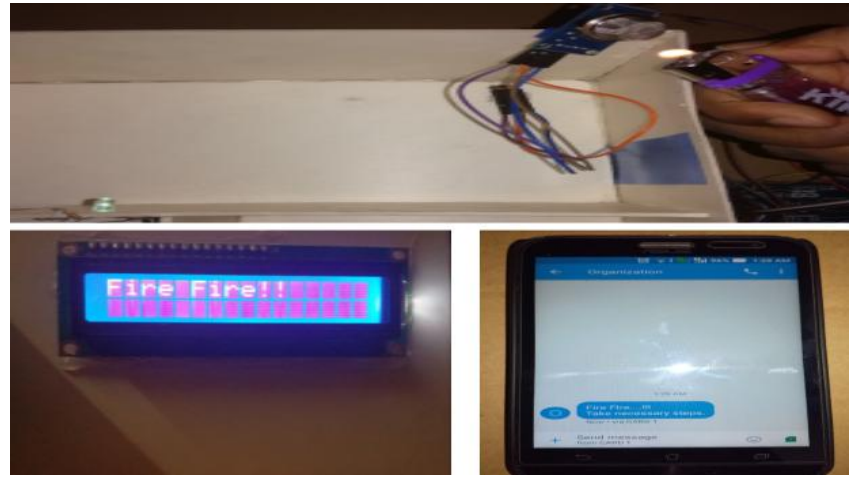

Figure 12: Live demo-gas/fire detection

\subsection{Control for appliance}

As the following figure 13 shows the control panel and how the system will work from web browser and control of light of the organization.

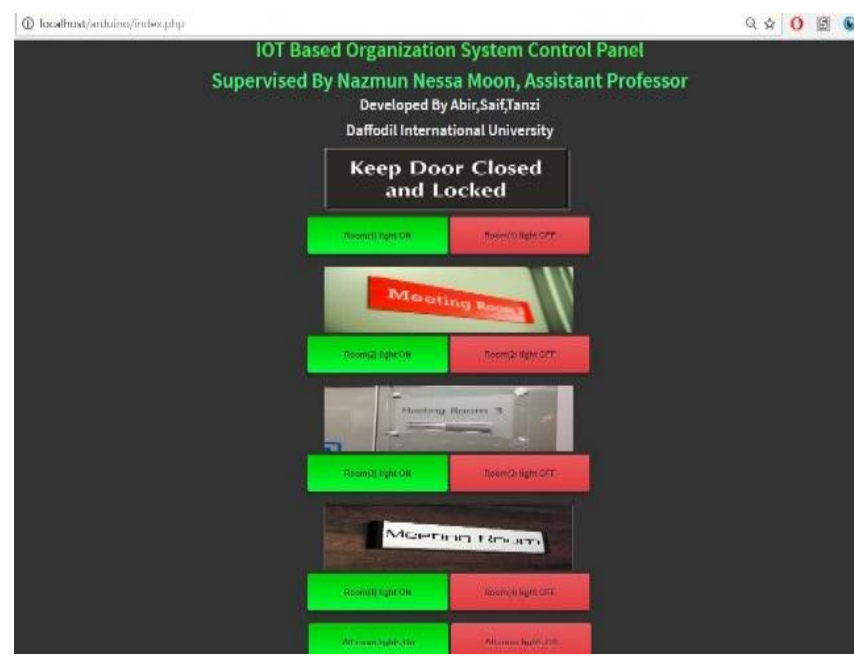

Figure 13: Control Panel Interface of Web Server

As the following figures 14 show the ARM voice control which is connected through Bluetooth module and when it commands "all light on" then all light of the organization will be "ON".

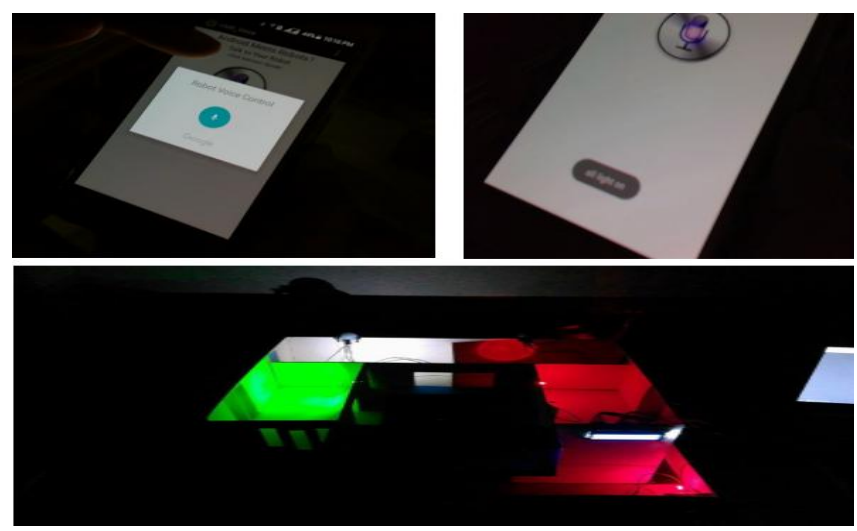

Figure 14: ARM voice Control Command

\section{APPLICATIONS}

There are huge amount of devices connected by the internet and sensors. Following could be a sample list of things that are supported the internet of Things.

- Organizations security system

- Hospital and Labs
- Home

- Electronic toll collection system.

- Monitoring and controlling of railway tracks, ship tracking systems.

- Entertainment and home security devices.

- Smart traffic control or vehicle control system.

\section{CONCLUSION}

We are developing an industrial system using internet of things technology. We are intended to produce an application near future for the observation of industrial appliance. We aim to serve as an efficient backbone for achieving a network of sensors and actuators which may facilitate for improving the performances of the day to day gadgets/activities for industry use.

\subsection{Challenges to overcome}

There are some limitation of our system beside lots of advantage. The connection of the wire is pretty much challenging and sometimes the sensors are not working properly due to its less ability. The maintenance of the voltage for every sensor is pretty much challenging because all the sensors don't need exact volt. In requirement base every sensors demands different voltage. Internet Connection is fluctuating which may create problems. SMS Alerts has to send but may have range problem. Decision making is difficult as this is question of many life \& industry. Wrong components selection for development

\subsection{Future scope}

The proposed system can be enhanced via

- Online server.

- GSM (Call, GPRS).

- Video recording/ surveillance.

- Implement a temperature sensor to maintain organization temperature and so on.

\section{ACKNOWLEDGMENT}

We acknowledge the advice of our beloved professors \& lecturers and those who previously worked on this concept. We also acknowledge the efforts of the critics of the journal for the modifications and suggestions to improve the quality of the paper.

\section{REFERENCES}

[1] D. Giusto, A. Iera, G. Morabito, L. Atzori (Eds.), The Internet of Things, Springer, 2010. ISBN: 978-1-4419$1673-0$.

[2] Li Da Zu" Internet of Things in Industries: A Survey" IEEE Transactions on Industrial Informatics, vol. 10, no. 4, November 2014

[3] N. Sklavos, P. Kitsos, O. Koufopavlou, "VLSI Design and Implementation of Homophonic Security System", proceedings of IEEE Computer Society Annual Symposium on VLSI (IEEE ISVLSI'12), Amherst, USA, August 19-21, 2012

[4] G. Gu and G. Peng, "The survey of GSM wireless communication system," in Proc. 2010 International Conference on Computer and Information Application (ICCIA), Tianjin, pp.121 - 124 .

[5] G. Mingming, Shaoliangshan, Huixiaowei, and Sunqingwei, "The System of Wireless Smart House Based on GSM and ZigBee," in Proc. 2010 International 
Conference on Intelligent Computation Technology and Automation (ICICTA), Changsha, pp.1017 - 1020.

[6] C. Felix and I. J. Raglend, "Home automation using GSM," in Proc. 2011 International Conference on Signal Processing, Communication, Computing and Networking Technologies (ICSCCN), Thuckafay, pp.15-19.

[7] C. Peijiang and J. Xuehua, "Design and Implementation of Remote Monitoring System Based on GSM," in Proc. 2008 Pacific-Asia Workshop on Computational Intelligence and Industrial Application (PACIIA '08), Wuhan, pp.678 - 681 .

[8] B. S. Rao, S. D. V. Prasad, and R.M. Mohan, "A prototype for Home Automation using GSM technology," in Proc. 2010 International Conference on Power, Control and Embedded Systems (ICPCES), Allahabad, pp.1-4.

[9] Rozita Teymourzadeh, CEng, Member IEEE/IET, Salah Addin Ahmed, Kok Wai Chan, and Mok Vee Hoong Faculty of Engineering, Technology \& Built Environment UCSI University Kuala Lumpur, Malaysia "Smart GSM based Home Automation System", 2013 IEEE Conference on Systems, Process \& Control (ICSPC2013), 13 - 15 December 2013.

[10] R. Llamas, R Reith, M. Shiere, "Apple Cedes Market Share in Smartphone Operating System Market as Android Surges and Windows Phone Gains, According to IDC", 7th August 2013, IDC Press Release, http://www.idc.com/getdoc.jsp?containerId=prUS242574 13.

[11] Mahesh N. Jivani, "GSM Based Home Automation System Using App-Inventor for Android Mobile Phone", International Journal of Advanced Research in Electrical, Electronics and Instrumentation Engineering (An ISO 3297: 2007 Certified Organization) Vol. 3, Issue 9, September 2014.

[12] Ming Yan, Hao Shi, "Smart Living Using Bluetoothbased Android Smartphone", International Journal of Wireless \& Mobile Networks (IJWMN) Vol.5, No. 1.

[13] B. Murali Krishna, V. Narasimaha Nayak, K. Ravi Kishore Reddy, B. Rakesh, P. Manoj Kumar, N.Sandhya, "Bluetooth Based Wireless Home Automation System Using FPGA", Journal of Theoretical and Applied Information Technology, 31st July 2015. Vol.77. No.3

[14] Nathan David, Abafor Chima, Aronu Ugochukwu, Edoga Obinna, "Design of a Home Automation System Using Arduino", International Journal of Scientific \& Engineering Research, Volume 6, Issue 6, June-2015
[15] Sirisilla Manohar, D. Mahesh Kumar, "E-Mail Interactive Home Automation System", International Journal of Computer Science and Mobile Computing, IJCSMC, Vol. 4, Issue. 7, July 2015, pg.78 - 87.

[16] Prof. R.S. Suryavanshi, Kunal Khivensara, and Gulam Hussain, Nitish Bansal, Vikash Kumar" Home Automation System Using Android and WiFi", International Journal of Engineering and Computer Science ISSN: 2319-7242 Volume 3 Issue 10 October, 2014.

[17] Poonam B. Patil, Rupali R. Patil, Swati V.Patil , Avadhoot R.Telepatil," Automation System Using Android and Arduino Board", International Journal of Innovative Research in Science, Engineering and Technology (An ISO 3297: 2007 Certified Organization) Vol. 5, Issue 4, April 2016

[18] S. Anusha, M. Madhavi, R. Hemalatha, "HOME Automation using ATMEGA328 Microcontroller and Android Application", International Research Journal of Engineering and Technology, Volume: 02 Issue: 06, Sep2015.

[19] V. Persis Priyanka, DR. K. Sudhakar Reddy, "PIR Based Security Home Automation System with Exclusive Video Transmission", International Journal of Scientific Engineering and Technology Research, Vol.04, Issue.18, June-2015

[20] S. M. Anamul Haque, S. M. Kamruzzaman and Md. Ashraful Islam, "A System for Smart-Home Control of Appliances Based on Timer and Speech Interaction," Proceedings of 4th International Conference on Electrical Engineering, pp. 128-131, January, 2006.

[21] Amrutha S, Aravind S, Ansu Mathew, Swathy Sugathan, Rajasree R, and Priyalakshmi S,"Speech Recognition Based Wireless Automation of Home Loads- E Home", International Journal of Engineering Science and Innovative Technology (IJESIT) Volume 4, Issue 1, January 2015.

[22] Ullah, Mohammad Samawat, Fernaz Narin Nur, and Nazmun Nessa Moon. "Optimization of wireless ad-hoc networks using an adjacent collaborative directional MAC (ACDM) protocol." International Journal of Computer Applications 114.3 (2015).

[23] Nur, F.N., Moon, N.N. Health care system based on cloud computing. Asian Transactions on Computers 2012; 2:9-11. 